\title{
A heuristic method for simultaneous tower and pattern-free field optimization on solar power systems
}

\author{
E. Carrizosa ${ }^{\mathrm{a}, \mathrm{b}}$, C. Domínguez-Bravo ${ }^{\mathrm{b}, *}$, E. Fernández-Cara ${ }^{\mathrm{b}, \mathrm{c}}$, M. Quero ${ }^{\mathrm{d}}$ \\ ${ }^{a}$ Department of Statistics and Operations Research, University of Seville, Spain \\ ${ }^{b}$ Mathematics Institute of the University of Seville, Spain \\ ${ }^{c}$ Department of Partial equations and Numerical analysis, University of Seville, Spain \\ ${ }^{d}$ Abengoa Solar New Technologies, Seville, Spain
}

\begin{abstract}
A heuristic method for optimizing a solar power tower system is proposed, in which both heliostat field (heliostat locations and number) and the tower (tower height and receiver size) are simultaneously considered.

Maximizing the thermal energy collected per unit cost leads to a difficult optimization problem due to its characteristics: it has a nonconvex black-box objective function with computationally expensive evaluation and nonconvex constraints.

The proposed method sequentially optimizes the field layout for a given tower configuration and then, the tower design is optimized for the previously obtained field layout. A greedy-based heuristic algorithm is presented to address the heliostat location problem. This algorithm follows a pattern-free method. The only constraints to be considered are: the field region and the nonconvex constraints
\end{abstract}

${ }^{*}$ Corresponding author.

Instituto de Matemáticas de la Universidad de Sevilla (IMUS)

Edificio Celestino Mutis- 1 Planta- D10.5

Avda. Reina Mercedes, s/n, 41012 Sevilla, Spain.

Tel: +34955420870 .

Email address: carmenanadb@us.es (C. Domínguez-Bravo) 
(which allow heliostats to not collide).

The absence of a geometrical pattern to design the field and the simultaneous optimization of the field and the tower designs make this approach different from the existing ones. Our method is compared against other proposals in the literature of heliostat field optimization.

Keywords: solar thermal power, heliostat field layout, thermo-economic optimization, nonconvex optimization, greedy algorithm

\section{Introduction}

Solar Power Tower (SPT) systems are known as one of the most promising technologies for producing solar electricity due to the high temperatures reached, resulting in high thermodynamic performances. For simplicity, we consider in this article an SPT system to consist of two elements: a tower and a field of (hundreds or thousands) of heliostats. The study of other related problems, see Section 5, are beyond the scope of this article.

In an SPT system direct solar irradiance is reflected by the heliostat field and concentrated onto a receiver placed at the top of the tower. In the receiver, this thermal energy at a high temperature is then transferred to the heat transfer fluid to produce electricity through a conventional thermodynamic cycle. The heliostat field is a group of mirrors having two-axis movement to reflect the direct light from the sun to the target point on the receiver aperture. The heliostat locations take into account the typical solar irradiance at the site.

The optimal design of an SPT system consists of determining the tower height, the shape and dimensions of the receiver aperture in the tower (Tower 
Optimization) and the location and number of the heliostats (Field Optimization) so as to optimize the annual thermal energy collected and the cost of the system. From the mathematical point of view, we want to simultaneously optimize several criteria. These objectives are in conflict to each other and they are usually aggregated into a single criterion, namely, the thermal energy collected per unit cost, see Ramos \& Ramos (2012); Spelling et al. (2012).

Three challenging issues are the dimensionality of the field optimization problem, with (a priori unknown) hundreds or thousands of variables, the nonconvex constraints related to the location of heliostats (which prevent the heliostats from colliding), and the evaluation of the objective function. This evaluation is implicitly defined by the subroutine, and due to the nature of the process, is not smooth, multimodal and has no apparent mathematical structure which can help to choose an appropriate optimization algorithm.

This optimization problem has great interest in the renewable energy literature, attracting researchers over the past thirty years. The problem continues being a very active research field, as can be appreciated in some reviews Lipps \& Vant-Hull (1978); Mills (2004); Romero et al. (2002); Stine \& Harrigan (2001); Behar et al. (2013).

Fixed geometrical patterns are traditionally used to solve the Field $O p$ timization problem. That is, the heliostat positions are given by some parameterized curves. The parameters are optimized in order to obtain a field layout. For instance, radially-staggered layouts are commonly assumed, originally proposed in Lipps \& Vant-Hull (1978), see also Collado (2009); Siala \& Elayeb (2001); Wei et al. (2010). This pattern consists on concentric semi- 
circumferences where the parameters indicate the separation between the circumferences and the angular distances between the heliostats located at the same circumference. The Spiral pattern is also used, where two parameters are optimized, see Noone et al. (2012).

Radially-staggered pattern has been so far the most popular SPT systems design. By the pattern itself, access is guaranteed to all heliostats in the field for cleaning or repairing work, since roads are naturally given. Although pattern-free fields do not define roads in their layout, (see e.g. the recently built Ivanpah system BrightSource (2014)), one may impose, as we do in this paper, heliostats to be sufficiently apart from each other, so that access to all heliostats in the field is possible. This would not be needed if new strategies for cleaning the heliostats were developed, see Alon et al. (2014).

Although these geometric patterns strongly simplify the Field Optimization problem, they may not reach good results if, for instance, time asymmetric weather conditions or terrain constraints are involved, as pointed out in Romero et al. (2002). The adaptability of the pattern is very limited and dependent on the chosen geometry, usually field symmetry is induced by the pattern itself.

Fixed-pattern strategies consider the number of heliostats to be located not given in advance: an oversized field (i.e., a field with a sufficiently large number of heliostats), is built, and then those heliostats reflecting less solar energy into the receiver are sequentially removed while guaranteeing that a given receiver outlet thermal power is attained. This way, although the optimal parameters for the oversized field were obtained, there is a high risk that a strong distortion exists between the original and final fields. 
We present a heliostats location procedure which will not force to follow a specific geometrical pattern, and, instead, will be a pattern-free optimization strategy. With our algorithm an initial oversized field is not needed, the final number of heliostats is found during the optimization process. A possible drawback is that road design and building may be more expensive. Contrarily, pattern-free fields are much more flexible and can be adapted (as will be shown in Section 4.3) to many geographical circumstances.

Most articles in the literature focus on the Field Optimization problem, see Collado (2009); Sánchez \& Romero (2006); Siala \& Elayeb (2001), or on the Tower Optimization separately, see Ghobeity \& Mitsos (2012). References to simultaneous optimization of the Field and Tower are very scarce. Pitz-Paal et al. (2011) and Ramos \& Ramos (2012) address the joint optimization by using a metaheuristic (genetic algorithm and simplex NelderMead) improved by local searches (Powell algorithm), always under the assumption of a radially-staggered pattern for the field.

This article presents a pattern-free procedure for the field layout optimization, and an optimization algorithm including the optimization of both the Tower and the Field.

The rest of the article is organized as follows. In Section 2, we describe the main ingredients affecting the performance of the SPT system. In Section 3 , our methodology to solve the problem is explained. In Section 4, we apply the optimization algorithms and analysis tools to a typical SPT design, and finally, in Section 5, our main results are summarized and some perspectives for further work are presented. 


\section{Problem statement}

In this Section, the SPT system, the variables used in the optimization process and the constraints that have to be satisfied are described. Finally, the two criteria involved in the objective function (energy and cost) and the optimization problem are presented.

\subsection{Decision variables}

Two types of decision variables appear, some associated to the height of the tower and the receiver aperture, and the remaining ones associated to the heliostats locations.

We will assume that the receiver consists of a cylinder pointing to the North, as can be seen in Figure 1 and is also explained in Behar et al. (2013); Collado (2008); Stine \& Harrigan (2001), among others. The front surface of the receiver, also known as the aperture, is especially important because it is here where strong radiative losses occur. For simplicity, only the two most relevant variables associated to the tower and the receiver design are considered, namely the aperture size, given by its radius $r_{a}$, and its height $h$ in the tower.

In what concerns the heliostat field, the heliostats locations, given by the coordinates $(x, y)$ of their centers, are the variables to be used. A heliostat is characterized by its geometry and its optical properties. All heliostats are assumed to be rectangular, to have the same dimensions and to be composed of rectangular facets.

From now on we will denote by $\Theta$ the variables related to the Tower, i.e. $\Theta=\left(r_{a}, h\right)$, and by $\mathcal{S}$ the finite set of coordinates of the centers of 


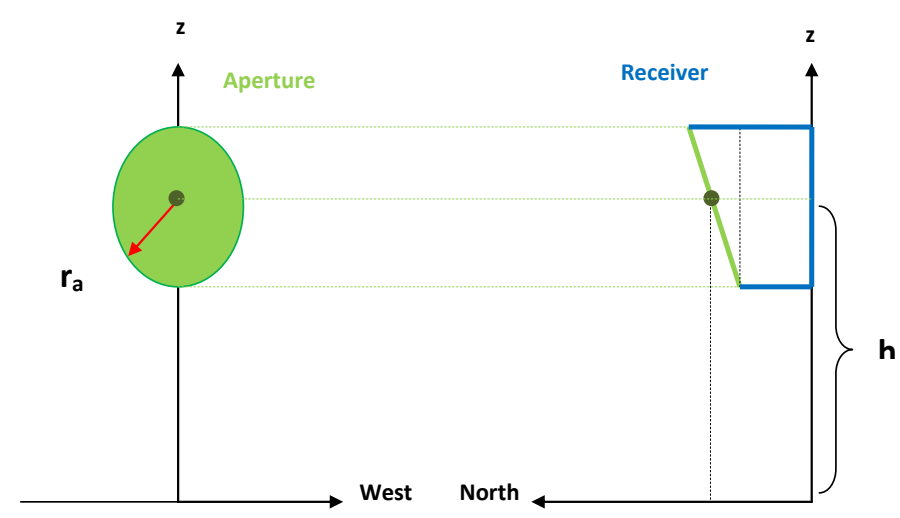

Figure 1: Receiver with circular Aperture

the heliostats that define the Field. The decision variables are $\Theta$ and $S$. Observe that the points of $\mathcal{S}$ belong to $\mathbb{R}^{2}$ and $\mathcal{S}$ can be viewed as a set of non-fixed cardinality. Consequently, we write in the sequel relations of the form $\mathcal{S} \subset \mathbb{R}^{2}$.

\subsection{Constraints}

Let $\Pi_{t}(\Theta, \mathcal{S})$ denote the receiver outlet thermal power at time $t$ for an SPT system with parameters $(\Theta, \mathcal{S})$. Usually, when designing an SPT system, a fixed instant of time is used to size the system, as explained in Collado (2008); Sánchez \& Romero (2006); Sanz-Bermejo et al. (2014). This time instant is known in the literature as the design point, $T_{d}$. At $T_{d}$, a minimal power $\Pi_{0}$ has to be achieved, that is: 


$$
\Pi_{T_{d}}(\Theta, \mathcal{S}) \geq \Pi_{0} .
$$

Other constraints on the variables related to the receiver are determined by the operation scheme of the system, which is in turn influenced by technical and legal regulations, leading to a compact set $\Theta$ as the feasible region for $\Theta$. There exist minimum and maximum values, $r_{\min }$ and $r_{\max }$ respectively, for the aperture radius and a maximum value $h_{\max }$ for the tower height. The feasible region $\Theta$ can be written as follows:

$$
\Theta=\left\{\left(r_{a}, h\right): r_{\min } \leq r_{a} \leq \min \left(h, r_{\max }\right) \leq h_{\max }\right\} .
$$

Related to the heliostat field we have to consider different constraints. The heliostats must be located within a given region $\Omega \subset \mathbb{R}^{2}$ and they have to rotate freely avoiding collisions between them. The feasible region $\mathscr{S}$ can be written as follows:

$$
\mathscr{S}=\left\{\begin{array}{lll}
\mathcal{S} \subset \Omega \subset \mathbb{R}^{2}: & |\mathcal{S}|<+\infty & \\
& \left\|(x, y)-\left(x^{\prime}, y^{\prime}\right)\right\| \geq \delta & \forall(x, y),\left(x^{\prime}, y^{\prime}\right) \in \mathcal{S} \\
& (x, y) \neq\left(x^{\prime}, y^{\prime}\right)
\end{array}\right\},
$$

where $\delta>0$ is a given positive parameter called security distance. This security distance has to be considered forcing the heliostats not to overlap.

\subsection{Functions}

Two criteria are taken into account for the optimization of the SPT system: the total investment cost and the annual thermal energy collected.

The cost function $C$ takes into account the investment in SPT system equipment (tower, receiver and heliostats), land and civil engineering costs. 
Hence, it depends on the receiver variables $\Theta$, that is, its radius $r_{a}$ and its height $h$, and the number of heliostats $|\mathcal{S}|$ in the field:

$$
C(\Theta,|\mathcal{S}|)=\beta_{1}(h+\kappa)^{\sigma}+\beta_{2} \pi r_{a}^{2}+c_{F}+c|\mathcal{S}|,
$$

where $\beta_{1}$ and $\beta_{2}$ are empirical constants with values in $(0,1), \kappa$ and $\sigma$ are positive and given by appropriate physical considerations and $c$ denotes the cost per heliostat. For simplicity, the cost associated with the land (purchasing and preparing) is considered fixed and denoted by $c_{F}$.

The collected annual thermal energy function $E$ takes the form:

$$
E(\Theta, \mathcal{S})=\int_{0}^{T} \tilde{\Pi}_{t}(\Theta, \mathcal{S}) d t-\gamma_{1},
$$

where the function $\tilde{\Pi}_{t}$ denotes the polynomial fitting of the receiver outlet thermal power at each time instant $t$ and $\gamma_{1}$ is a constant that mesures the fixed thermal energy losses related to the whole system.

The receiver outlet thermal power value at time $t$ is calculated by adding the values collected into the receiver by each heliostat and written as follows:

$$
\Pi_{t}(\Theta, \mathcal{S})=I(t) f_{\text {ref }} \sum_{(x, y) \in \mathcal{S}} \varphi(t, x, y, \mathcal{S}, \Theta)-\gamma_{2} \pi r_{a}^{2} .
$$

Here $I(t)$ is the so-called instantaneous direct solar irradiance, $f_{r e f}$ is the heliostat reflectance factor, $\varphi$ is the product of the efficiency factors (usual in this framework), that is, $\varphi=f_{c o s} \cdot f_{s b} \cdot f_{s p} \cdot f_{a t}$ and the constant $\gamma_{2}$ is related with the thermal energy losses associated to the receiver size.

In particular, $f_{\cos }=f_{\cos }(t, x, y, \Theta)$ is the cosine efficiency, see Collado \& Turégano (1989); $f_{s b}=f_{s b}(t, x, y, \mathcal{S}, \Theta)$ is the shading and blocking efficiency, 
see Sassi (1983); Collado \& Guallar (2012); Stine \& Harrigan (2001); $f_{s p}=$ $f_{s p}(t, x, y, \Theta)$ is the interception efficiency or spillage factor, see Collado \& Turégano (1986) and finally, $f_{a t}=f_{a t}(x, y)$ is the atmospheric efficiency, which takes into account atmospheric losses between the heliostat and the receiver, see Biggs \& Vittitoe (1976); Collado \& Turégano (1989).

Regarding the annual thermal energy function, the polynomial fitting is performed using a discretization over the year considering different time steps. Due to the complexity of the model, it seem very hard to measure in a rigorous way the error caused by such discretization. However, we can have an idea by checking how stable results are with respect to the grid used. For instance, if we consider 5, 9 or 13 different hours to compute the daily thermal energy, for different fixed days we obtain the results shown in Table 1 . These results suggest that 1 or 2 decimal digits are acceptable and significant.

\begin{tabular}{|c|c|c|c|c|}
\hline $\mathrm{E}$ (MWth) & March & June & September & December \\
\hline$E_{5}$ & 0.371825 & 0.405744 & $0 . \mathbf{3 7 4} 4933$ & 0.272064 \\
\hline$E_{9}$ & 0.367207 & 0.406332 & $0 . \mathbf{3 7 1 7 3 3}$ & 0.268578 \\
\hline$E_{13}$ & 0.364203 & 0.410110 & 0.370317 & $0 . \mathbf{2} 44562$ \\
\hline
\end{tabular}

Table 1: Daily Thermal Energy

The annual thermal energy collected is computed using an algorithm similar to NSPOC procedure, that is described in Crespo \& Ramos (2009). We refer the reader to Biggs \& Vittitoe (1976); Collado \& Turégano (1986, 1989); 
Stine \& Harrigan (2001), for further details.

\subsection{Optimization problem}

As mentioned above, the two criteria involved are the cost of the system and the annual thermal energy collected. No common optimum can be found for both criteria, so they are aggregated into one single objective, namely, the maximization of annual thermal energy collected per unit cost. Written this way, the optimization problem we are addressing is the following:

$$
(\mathcal{P}) \begin{cases}\max _{\Theta, \mathcal{S}} & F(\Theta, \mathcal{S})=E(\Theta, \mathcal{S}) / C(\Theta,|\mathcal{S}|) \\ \text { subject to } & \Theta \in \Theta \\ & \mathcal{S} \in \mathscr{S} \\ & \Pi_{T_{d}}(\Theta, \mathcal{S}) \geq \Pi_{0}\end{cases}
$$

\section{An alternating procedure to design the SPT system}

In order to solve $(\mathcal{P})$, an alternating procedure is suggested, in which one sequentially optimizes the field layout for a given tower design and then, the tower design is optimized for the previously obtained field. In other words, we alternately solve $\left(\mathcal{P}_{\Theta}\right)$ and $\left(\mathcal{P}_{\mathcal{S}}\right)$. Both subproblems are described below. Thus, the optimization problem $(\mathcal{P})$ has two blocks of decision variables, namely those related to the design of the tower $\Theta$, and the coordinates of the centers of the heliostats $\mathcal{S}$, related to the heliostat field.

A drawback of the alternating strategy is that starting with a bad solution the algorithm could converge to inefficient solutions. This risk can be prevented with a multistart of the alternating process, considering different 
random initial tower configurations as initial solutions. Although we do not have technical proofs, we have empirical evidences which show that the algorithm converges to solutions outperforming heliostat fields such as PS10, RPS10 and Spiral based, see Section 4.

The $\left(\mathcal{P}_{\Theta}\right)$ subproblem, that is, the optimization of the heliostat field for a fixed tower, can be written as follows:

$$
\left(\mathcal{P}_{\Theta}\right) \quad \Theta \text { fixed } \begin{cases}\max _{\mathcal{S}} & F(\Theta, \mathcal{S}) \\ \text { subject to } & \mathcal{S} \in \mathscr{S} \\ & \Pi_{T_{d}}(\Theta, \mathcal{S}) \geq \Pi_{0}\end{cases}
$$

Observe that the cardinality of $\mathcal{S}$ (number of heliostats) is not fixed in advance in problems $(\mathcal{P})$ and $\left(\mathcal{P}_{\Theta}\right)$, thus we cannot express them as standard optimization problems in fixed dimension.

The other subproblem $\left(\mathcal{P}_{\mathcal{S}}\right)$ given below describes the optimization of the tower and receiver when the field of heliostats $\mathcal{S}$ is fixed.

$$
\left(\mathcal{P}_{\mathcal{S}}\right) \quad \mathcal{S} \text { fixed } \begin{cases}\max _{\Theta} & F(\Theta, \mathcal{S}) \\ \text { subject to } & \Theta \in \Theta \\ & \Pi_{T_{d}}(\Theta, \mathcal{S}) \geq \Pi_{0}\end{cases}
$$

The alternating algorithm used to solve the optimization problem $(\mathcal{P})$ is described in Algorithm 1. As we have said, this algorithm alternatively solve the tower and the heliostat field optimization problems. We consider that the algorithm has performed a complete iteration when a tower problem and a field problem have been solved. Each time an optimization subproblem is solved, that is $\left(\mathcal{P}_{\mathcal{S}}\right)$ or $\left(\mathcal{P}_{\Theta}\right)$, the highest value obtained for the objective function and the system design associated to this value are stored in the 
variables $\Upsilon_{\text {objective }}$ and $\Upsilon_{\text {design }}$ respectively. At the end of each iteration, the relative error is calculated. If it is lower than a given value $\epsilon$ (positive and small), the algorithm stops and returns the highest computed value.

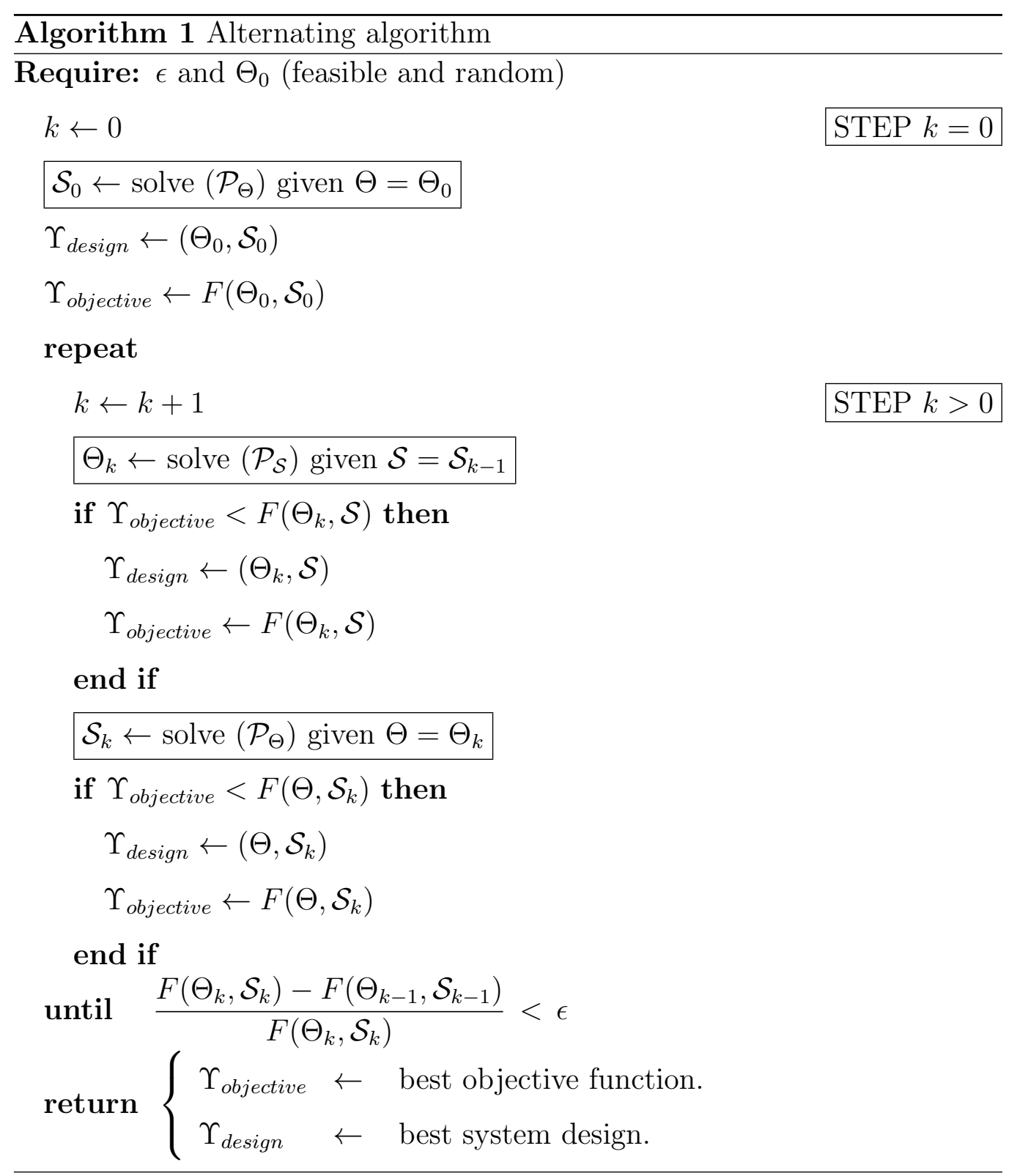


At the starting step, the initial value $\Theta_{0}$ for the variable $\Theta$ is set randomly in the feasible region $\Theta$. Once this initial value is calculated, the first heliostat field is obtained solving problem $\left(\mathcal{P}_{\Theta}\right)$, with $\Theta=\Theta_{0}$.

After the initial step is performed, both problems, $\left(\mathcal{P}_{\mathcal{S}}\right)$ and $\left(\mathcal{P}_{\Theta}\right)$ are solved at each iteration in this same order. In Subsection 3.1, we detail the steps in the optimization of the heliostat field for a fixed tower (the $\left(\mathcal{P}_{\Theta}\right)$ subproblem), since the other subproblem $\left(\mathcal{P}_{\mathcal{S}}\right)$ is directly solvable by standard techniques as explained below.

We suggest to optimize $\Theta$ by a cyclic coordinate method, see Bazaraa et al. (2006). We start with an initial random solution in the feasible region, in our case $\Theta$, at which the objective function is evaluated. The algorithm performs at each iteration two local searches using as search direction each coordinate axis. No difficulties are expected, since, in our experience, the function $F(\cdot, \mathcal{S})$ has a unimodal shape, shown in Figure 2 using the reference field layout PS10 given in Noone et al. (2012).

\subsection{Field optimization}

We are going to describe in this subsection an algorithm for solving problem $\left(\mathcal{P}_{\Theta}\right)$. Note that the number of variables (heliostats centers) is not fixed. Even fixing the number of heliostats, the high number of variables $(3,000$ in recent commercial plants Burgaleta et al. (2011)), together with the characteristics of the objective function (black-box, multimodal, non-smooth and high time consuming) make this problem difficult to solve.

As we have already mentioned, there exist many approaches considering fixed-patterns to locate the heliostats. In Sánchez \& Romero (2006), a greedy strategy is used to locate the heliostats in a fixed rectangular gridding. In a 


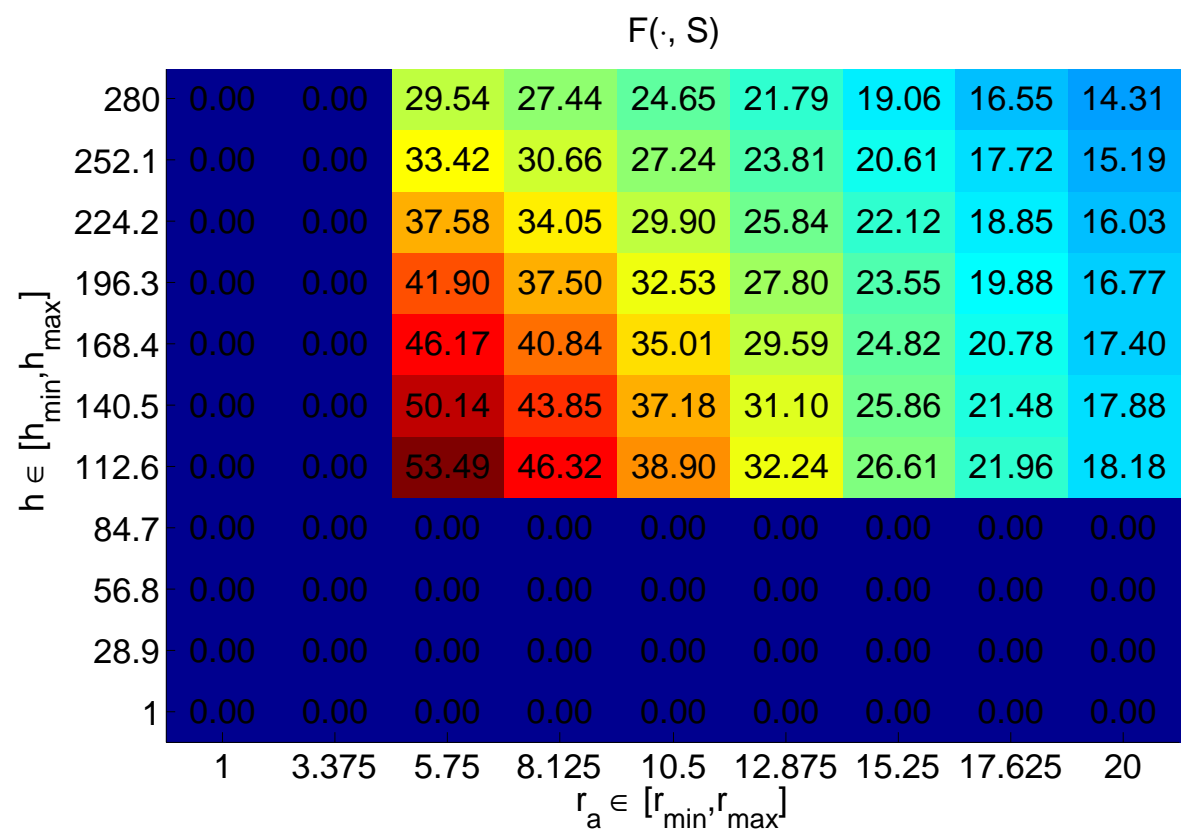

Figure 2: $F(\cdot, \mathcal{S})$ values fixed $\mathcal{S}=P S 10$ 
first step, the annual thermal energy at any point of the grid is calculated. The heliostats are located at the best positions of the grid. To prevent future heliostats from being located in the optical path of those previously placed, a mirror image of the blocking effect is added without considering overlapping. They introduce a penalizing weight factor to favor the location of heliostats closer to the tower, due to the need of increasing the heliostat density given by the grid in the best region of the field (see Figure 3(a)).

Our procedure also uses a greedy strategy although we do not impose any fixed configuration to locate the heliostats. The process we are going to present is different from others in the literature in three aspects:

- No parametric form is used as a field pattern (e.g. we do not assume that the field has to possess a radially staggered shape), therefore the strategy can be extended directly to complex field regions, see Section 4.3 .

- The number of heliostats is selected according to the thermal power requirements into the receiver, avoiding oversizing. This way computational time and distortion on the optimization results are removed.

- The procedure can be use to generate initial fields. The final result is susceptible of refinement with local improvements using the method presented in Buck (2014).

We present a greedy based algorithm which locates the heliostats one by one at the best feasible position, that is, the position where the annual thermal energy collected is highest for a given tower. The process is repeated until no improvement is reached in the annual thermal energy collected. The 
heliostats are located freely, without any pre-arranged distribution. Only two geometrical constraints have to be taken into account: the field shape constraint and the heliostat constraints to avoid collisions, see (3). At each step, the annual thermal energy collected into the receiver by each heliostat is modified due to the new shading and blocking effects that the heliostat is causing. This is the main reason of increase of the computing time. Once a new heliostat is located and the shading and blocking effects are incorporated, the process must be repeated.

Obviously, the first problem, $\left(\mathcal{P}_{\Theta}^{0}\right)$, involves locating the first heliostat center when only the field shape constraint is considered:

$$
\left(\mathcal{P}_{\Theta}^{0}\right) \quad \Theta \text { fixed } \begin{cases}\max _{(x, y)} & E(\Theta,\{(x, y)\}) \\ \text { subject to } & (x, y) \in \Omega\end{cases}
$$

This problem has an easy-to-handle objective function, as plotted in Figure 3(a). In return, when we have already located at least one heliostat in the field and we have obtained a field denoted by $\mathcal{S}_{k-1}^{*}$ with $k-1$ heliostats which respects the constraints, the problem $\left(\mathcal{P}_{\Theta}^{k}\right)$ described below is difficult to solve, since nonconvex constraints are involved and the energy function has a complex behavior due to the shading and blocking effects, see Figure 3(b). 


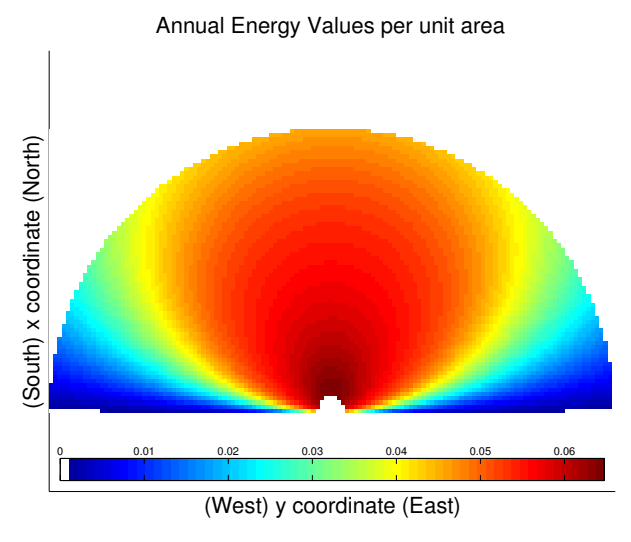

(a) $k=0$

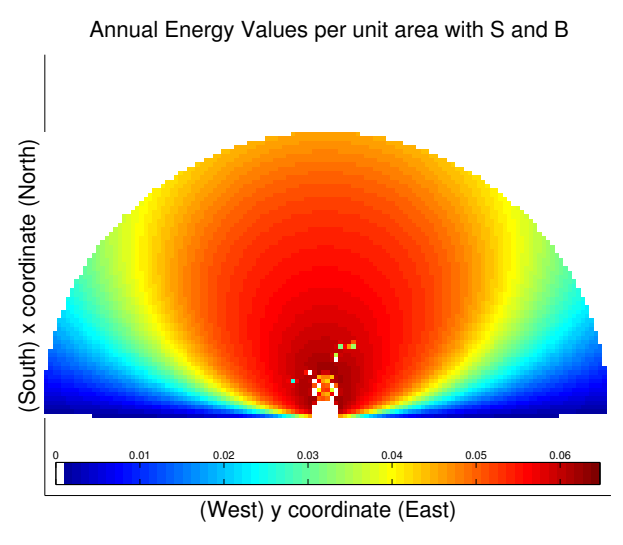

(b) $k=25$

Figure 3: Annual Thermal Energy collected

Let us introduce the notation $\mathcal{S}_{k}^{*}=\left\{\mathcal{S}_{k-1}^{*},(x, y)\right\}$, where $(x, y)$ denotes the variable with respect we maximize in problem $\left(\mathcal{P}_{\Theta}^{k}\right)$ :

$$
\left(\mathcal{P}_{\Theta}^{k}\right) \quad \Theta \text { fixed } \begin{cases}\max _{(x, y)} & E\left(\Theta,\left\{\mathcal{S}_{k-1}^{*},(x, y)\right\}\right) \\ \text { subject to } & (x, y) \in \Omega \\ & \left\|(x, y)-\left(x^{\prime}, y^{\prime}\right)\right\| \geq \delta_{0} \quad \forall\left(x^{\prime}, y^{\prime}\right) \in \mathcal{S}_{k-1}^{*} .\end{cases}
$$

As noticed the $k$-step the problem is equivalent to maximizing the annual thermal energy collected into the receiver by the new heliostat, because the cost function is fixed at each step. Note that, for simplicity, we assume that the heliostat cost is independent on its position in the field.

Now, we focus on the resolution of the problem $\left(\mathcal{P}_{\Theta}^{k}\right)$, i.e. the location of one heliostat, and we introduce the Algorithm 2 called Greedy Algorithm to solve it. It is well known that the energy function evaluation is computationally expensive, see Sánchez \& Romero (2006); that is why, in this algorithm, 
we approximate it by a much simpler function. Thus, instead of computing $E$ as in (5), the receiver outlet thermal power (6) at the design point $T_{d}$ is used; this is $\Pi_{T_{d}}$. More accurate approximations, as those suggested in Spelling et al. (2012); Zhang et al. (2007) based on calculating the receiver outlet thermal power at several time instants could be used, at the expense of increasing the already high computational cost.

Using $\Pi_{T_{d}}$ as the objective function leads to more compact fields. The reduction on the shading and blocking effects at this time instant favors closer heliostat positions. In order to compensate for this, the safety distance value is modified. The new safety distance $\delta_{0}$ is defined as the product of the initial safety distance $\delta$ and a new parameter $F_{\text {sep }}$ that can be in the range [1,2]. It depends on the selected design point, the heliostat size and the feasible region among others, that is why it has to be set for each problem.

The Greedy Algorithm considers $N_{\text {ini }}$ different random feasible initial solutions. This multistart procedure is used to avoid local minima. The final solution is selected according to the receiver outlet thermal power at the design point collected by each new field layout. 


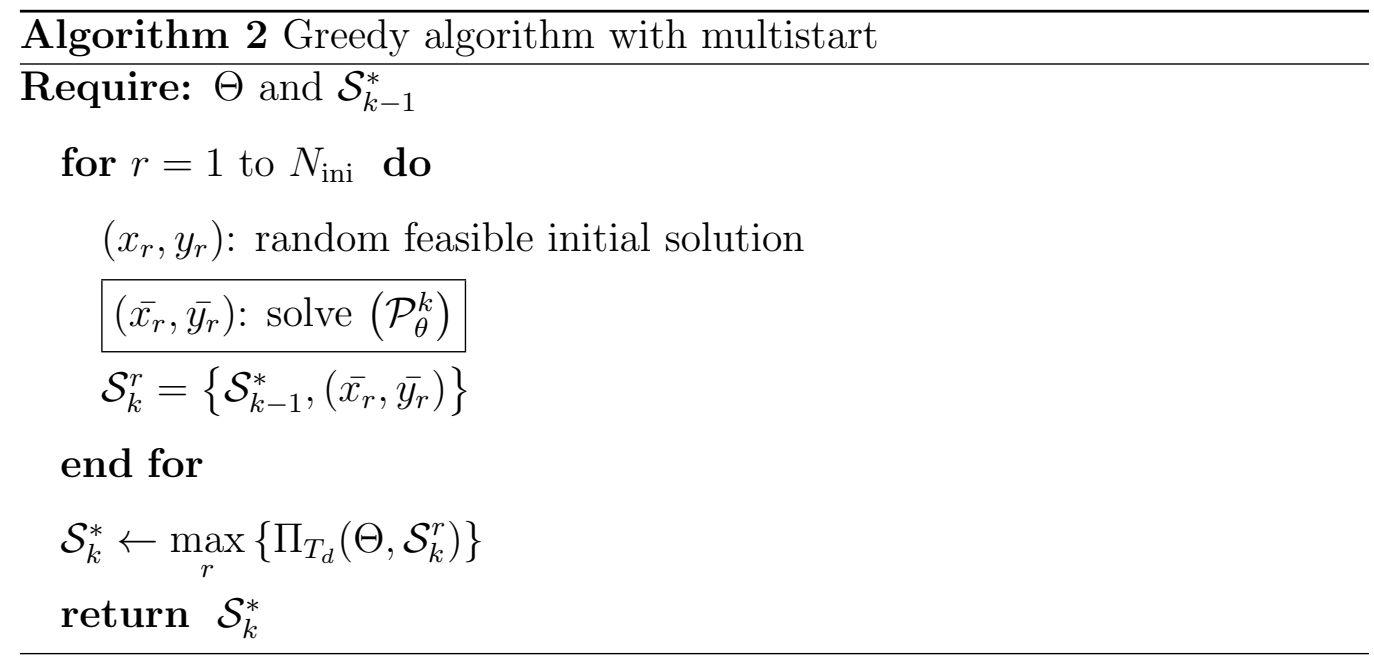

The complete field is generated using the Field Layout Algorithm which scheme is given in Algorithm 3 and described below. In order to solve problem $\left(\mathcal{P}_{\Theta}\right)$, since the functions involved are highly multimodal, and the output strongly depends on the starting points used on Algorithm 2, the complete process is repeated $N_{\text {sem }}$ times with different seed states. The initial data required are the fixed variables related with the tower size $\Theta$, the number of times that the algorithm will be repeated $N_{\mathrm{sem}}$, and the number of initial solution to be used in the greedy multistart procedure $N_{\text {ini }}^{*}$.

Algorithm 3 has two different steps. At first step $(k=0$, no heliostat in the field), there are not shading and blocking effects involving multimodality in the objective function. Therefore no multistart strategy is required, and the number of initial solutions is set to one. Once the first heliostat is located in the field $(k>0)$, the multimodality of the problem appears and $N_{\mathrm{ini}}^{*}$ different feasible random initial solution are considered. As we have already described, the heliostats are located solving $\left(\mathcal{P}_{\Theta}^{k}\right)$ using Algorithm 2, with the corresponding $N_{\text {ini }}$ value. 
When $k>0$, two phases of Algorithm 3 can be differentiate, Phase A and $B$. Phase A consists on locating heliostats using Algorithm 2 until the thermal power requirement $\Pi_{0}$ is reached. At the end of this phase we could stop locating heliostats, however we continue with Phase B applying a different stopping criterion. We continue locating heliostats in this phase provided that the system annual thermal energy collected per unit cost increases.

It may happen that there exist some manufacturer requirements on the receiver outlet thermal power collected by the field in order to prevent damages, see Buck et al. (2006). For this reason, Phase B should stop when a power upper limit, $\Pi_{1}$, is reached. In this case the heliostat field layout is stored in $\Upsilon_{\text {design-B1 }}$. However, this field may be improved and forgetting this thermal power limitation. The algorithm continues until the system annual thermal energy collected per unit cost does not increase. In this case, the solution are the highest annual value attained $\Upsilon_{\text {objective }}$ and its tower and field configuration, $\Upsilon_{\text {design }}$. 


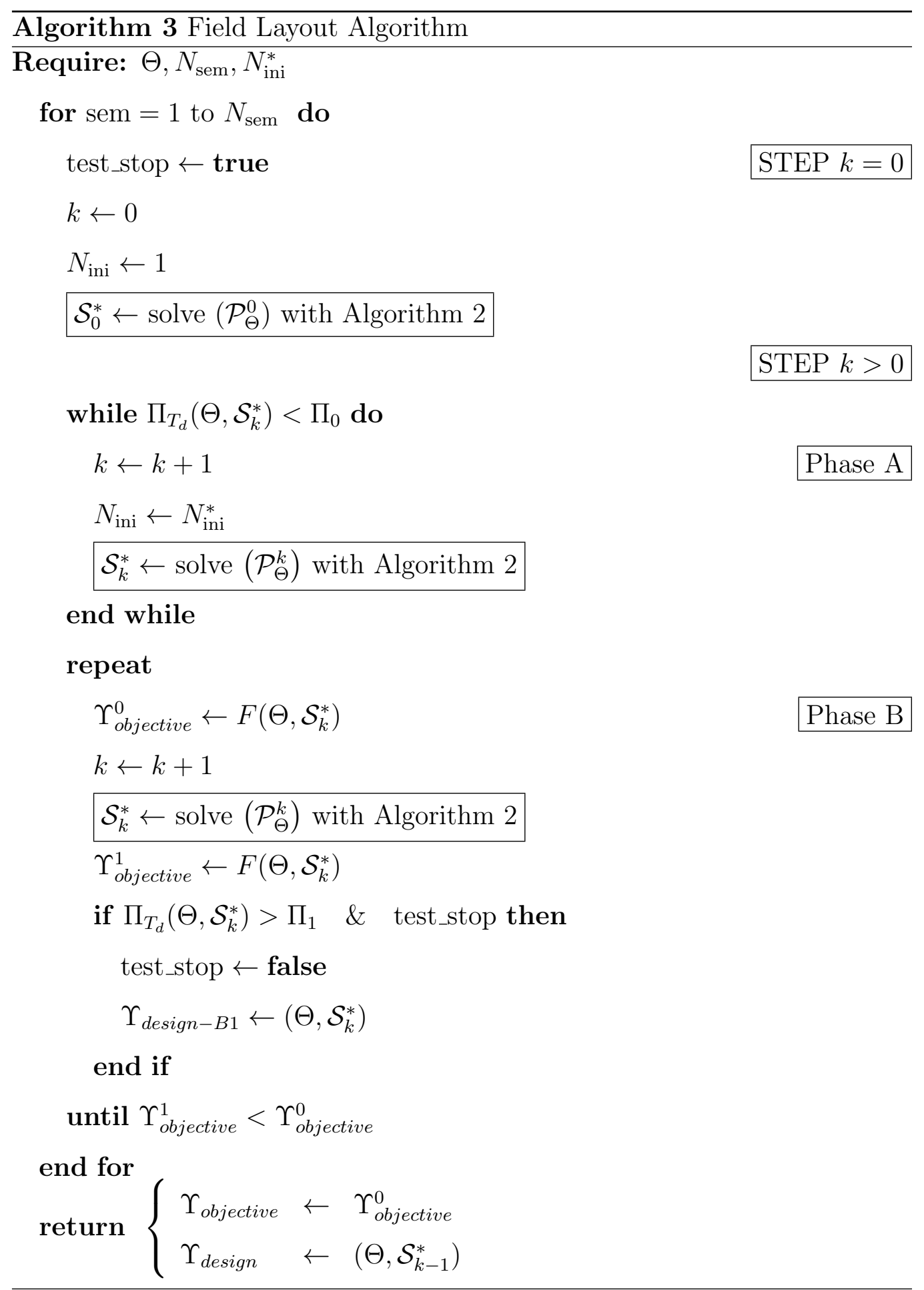


The proposed heuristic algorithm does not give an optimal solution, nor the ones proposed in the literature. Given the number of variables expected, we obtain a reasonable solution compared against the reference fields PS10 and RPS10.

Note that the proposed greedy algorithm locates the heliostats one by one. This strategy can be extended to locate $n$ heliostats in block, being $n$ small to maintain the numerical treatability of the problem.

\section{Results}

A prototype of the algorithms has been developed in Matlab ${ }^{\circledR}$, using fminbnd and fmincon as local search routines to solve the optimization subproblems involved solving $\left(\mathcal{P}_{\Theta}\right)$ and $\left(\mathcal{P}_{\mathcal{S}}\right)$ respectively.

The accuracy and feasibility of the results have been confirmed by comparing to the heliostat fields obtained with benchmark procedures. Although in the greedy optimization procedure an approximation of the objective function (based on the thermal power collected at the design point) is used. When comparing the results, the accurate thermal energy function is used (see in the tables column label with $E$ ). Three fields are considered for comparison

purpose, with a common tower design. Following Noone et al. (2012), such test fields are called here PS10, RPS10 (redefined PS10) and Spiral. The values of the geographical, physical and geometrical parameters are given in Table 2. 


\begin{tabular}{|c|c|c|}
\hline Parameter & Default value & Ref. \\
\hline \multicolumn{3}{|l|}{ Location and Time } \\
\hline Site & Sanlúcar la Mayor (Seville) & Osuna et al. (2004) \\
\hline Latitude & $37^{\circ} 26^{\prime} \mathrm{N}$ & Noone et al. (2012) \\
\hline Longitude & $6^{\circ} 15^{\prime} \mathrm{W}$ & Noone et al. (2012) \\
\hline Design Point $T_{d}$ & March Day 21 Hour 12 & assumed \\
\hline Design direct normal irradiance DNI & $823.9 \mathrm{~W} / \mathrm{m}^{2}$ & assumed \\
\hline DNI model & cloudless sky & assumed \\
\hline \multicolumn{3}{|l|}{ Receiver } \\
\hline Aperture tilt & $12.5^{\circ}$ & Noone et al. (2012) \\
\hline Receiver Technology & Saturated Steam & Osuna et al. (2004) \\
\hline \multicolumn{3}{|l|}{ Heliostat } \\
\hline Heliostat & Sanlucar 120 & Noone et al. (2012) \\
\hline Hel. width & $12.84 \mathrm{~m}$ & Noone et al. (2012) \\
\hline Hel. height & $9.45 \mathrm{~m}$ & Noone et al. (2012) \\
\hline Hel. optical height $z_{0}$ & $5.17 \mathrm{~m}$ & Osuna et al. (2004) \\
\hline Hel. minimal safety distance $\delta$ & heliostat diagonal & Buck (2014) \\
\hline$\sigma_{\text {optical }}$ & $2.9 \mathrm{mrad}$ & Noone et al. (2012) \\
\hline \multicolumn{3}{|l|}{ Field } \\
\hline Slope & $0^{\circ}$ & assumed \\
\hline Shape & semicircle & assumed \\
\hline Minimum radius & $50 \mathrm{~m}$ & assumed \\
\hline Maximum radius & $1,000 \mathrm{~m}$ & assumed \\
\hline Maximum surface & $156.68 h a$ & assumed \\
\hline
\end{tabular}

Table 2: Parameter Values 


\subsection{Heliostat field layouts comparison given a Tower-Receiver configuration}

PS10 as given in Noone et al. (2012), is an SPT system similar to a solar commercial plant located in Sanlúcar la Mayor, Spain, and it is one of the most popular test instances. The Tower-Receiver variables for the PS10 are fixed as parameters, assuming a tower height of $100.50 \mathrm{~m}$ and an aperture radius of $6.39 \mathrm{~m}$, that is $\Theta=(6.39,100.5)$. RPS10 is a variant suggested in Noone et al. (2012), in which a local search is performed, taking the PS10 layout as starting point, optimizing the parameters configuration of the system. Finally, Spiral, as introduced in Noone et al. (2012), is another field layout obtained when a spiral pattern is given for the field layout and the parameters of the spiral are optimized.

In Figure 4, the previous heliostat field layouts and also the GPS10 configurations, computed using our greedy algorithm, are shown with the same number of heliostats. This GPS10 configuration have been obtained after performing different experiments varying $F_{\text {sep }}$ with $N_{\text {sem }}=5$. The best configuration that we show is obtained with $F_{\text {sep }}=1.5$. 


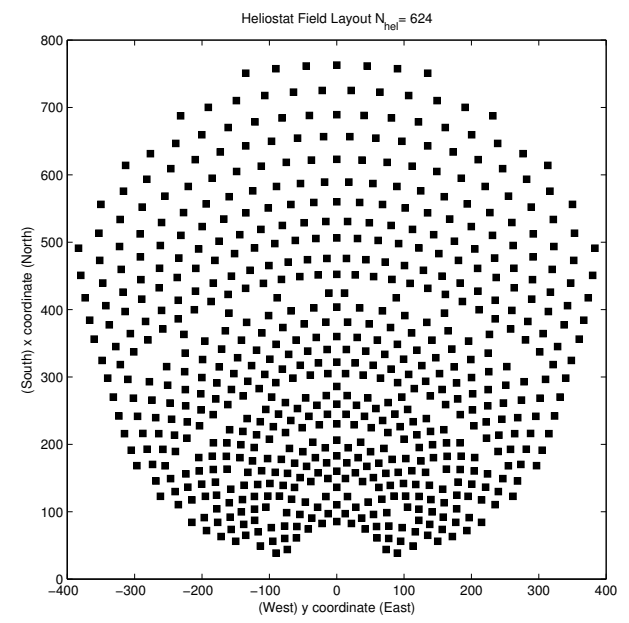

(a) PS10

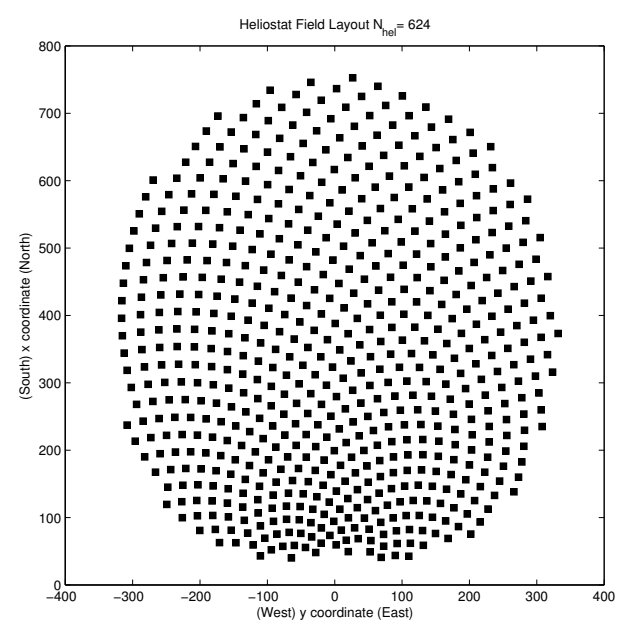

(c) Spiral

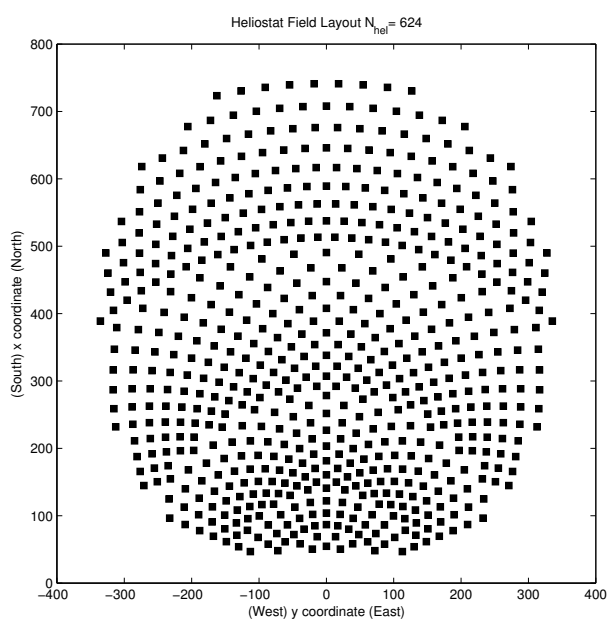

(b) RPS10

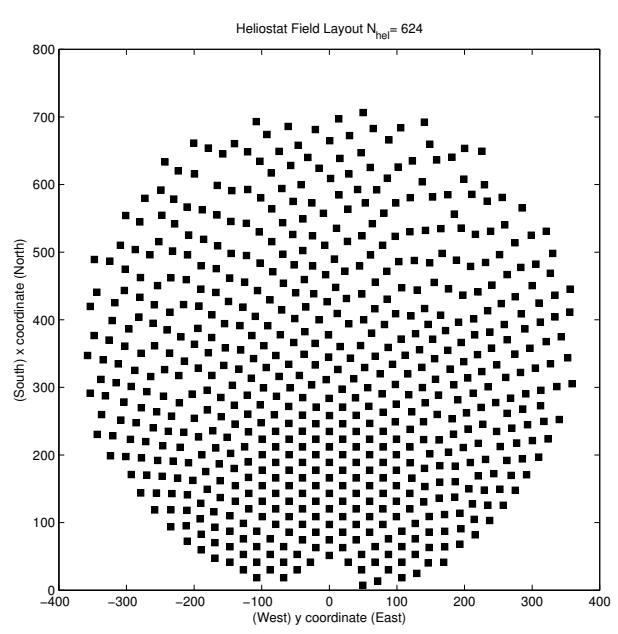

(d) GPS10 Phase A

Figure 4: Heliostat Field Layouts

The receiver outlet thermal energy at the design point $T_{d}$, the annual thermal energy collected and annual thermal energy collected per unit cost for each field layout, are given in Table 3. Note that, in view of the results in Table 1 , at best, we can expect that the computations are $1 \%$ accurate and 
this means that the differences of performance of PS10, RPS10 and Spiral are between the error of calculation. GPS10-(Phase B) results in higher annual energy collection per unit cost, which implies that a larger number of heliostats results in lower cost.

If we compare the results obtained fixing the same number of heliostats, GPS10-(Phase A), we can see that our algorithm provides similar results compared to PS10, RPS10 and Spiral regarding the annual thermal energy, and better results than Spiral regarding the receiver outlet thermal energy at $T_{d}$. Note that PS10 and Spiral fields are solutions of optimization problems in which a geometrical configuration pattern is imposed. However, the solutions so obtained, though optimal under the pattern based constraints, may be suboptimal if such constraints are removed.

\begin{tabular}{lcccc}
\hline Field & $N$ & $\Pi_{T_{d}}\left(\right.$ MWth $\left.10^{-2}\right)$ & $E\left(\right.$ GWHth $\left.10^{-3}\right)$ & $F$ \\
\hline PS10 & 624 & 0.43 & 0.12 & 0.50 \\
RPS10 & 624 & 0.43 & 0.12 & 0.50 \\
Spiral & 624 & 0.42 & 0.12 & 0.50 \\
GPS10 Phase A & 624 & 0.43 & 0.12 & 0.50 \\
GPS10 Phase B & $\mathbf{9 4 3}$ & $\mathbf{0 . 6 2}$ & $\mathbf{0 . 1 7}$ & $\mathbf{0 . 5 4}$ \\
\hline
\end{tabular}

Table 3: Thermal Power at $T_{d}$, Annual Thermal Energy and Annual Thermal Energy per unit Cost.

The RPS10 and GPS10 fields are shown in Figure 5 with the heliostats colored according to their contribution to the annual thermal energy collected. 


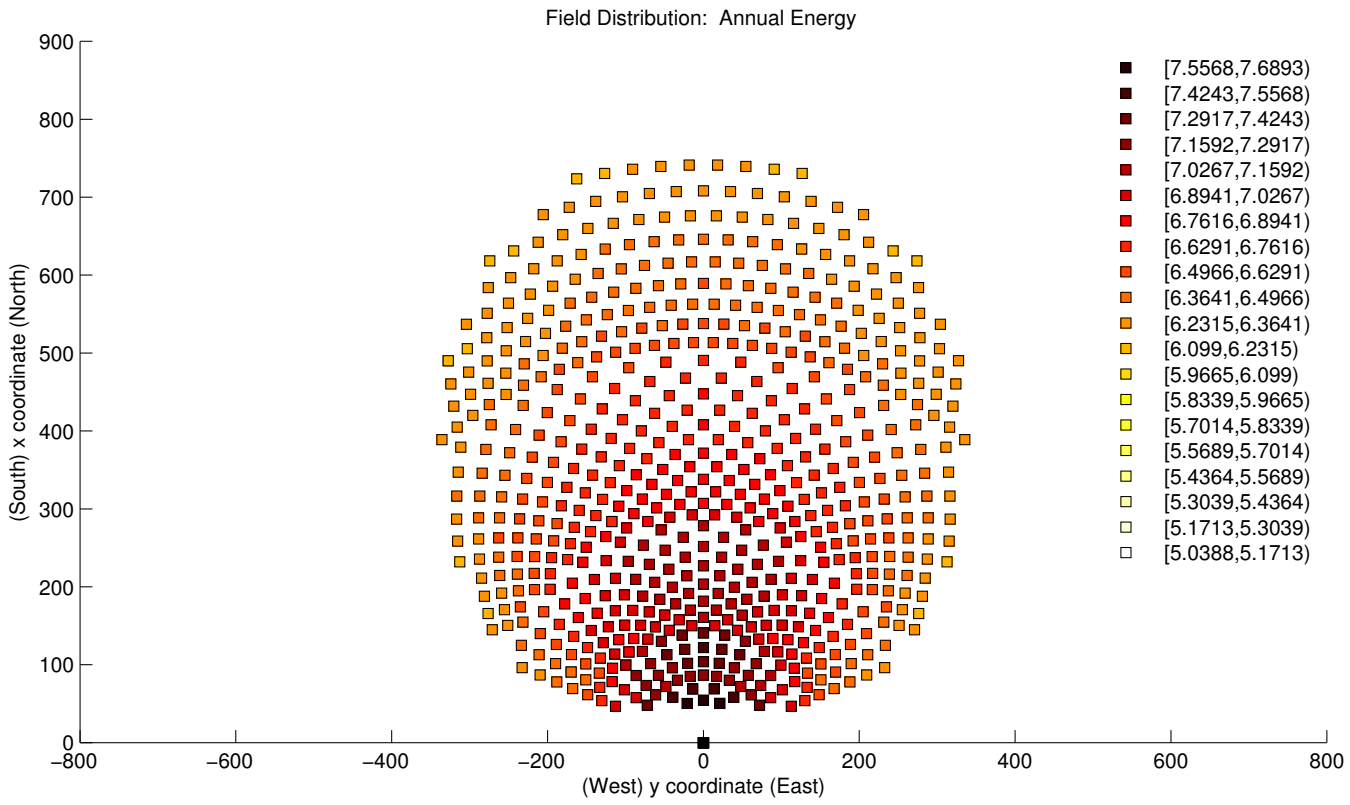

(a) RPS10

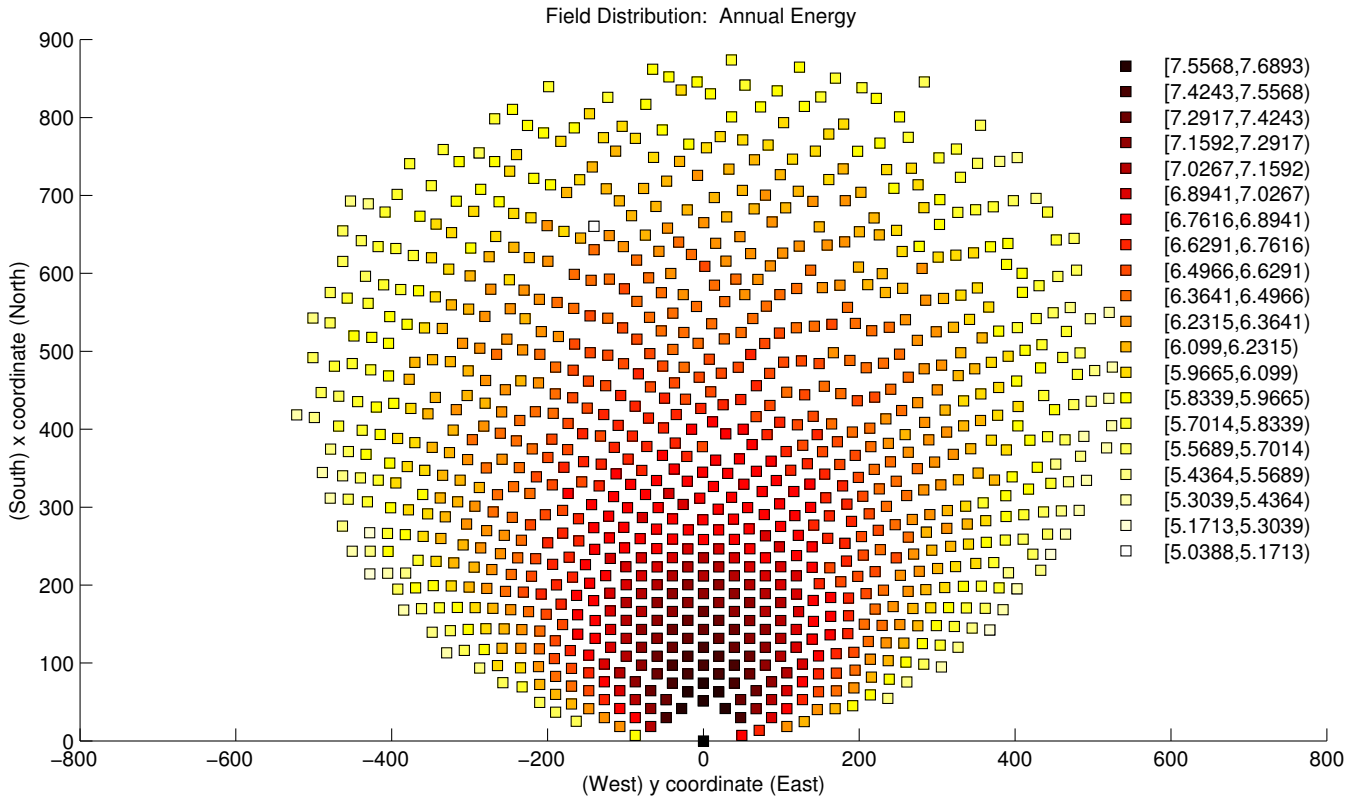

(b) GPS10 Phase B

Figure 5: Annual Thermal Energy collected per heliostat 
In order to compare the four fields layouts we have sorted the heliostats of each field in terms of the amount of annual thermal energy collected. Some results are described below, displaying the energy and the cost in terms of the amount of heliostats. The annual thermal energy collected versus the number of heliostats located in the field is shown in Figure 6(a); the cost of the SPT system versus the number of heliostats is depicted in Figure 6(b) and in Figure 6(c) is shown the objective function, that is, the annual thermal energy collected per unit cost versus the number of heliostat. In these three figures the four fields have the same behavior, but, when displaying the marginal annual thermal energy added by each heliostat in the field, see Figure 6(d), some differences can be observed due to the different heliostat location procedures. 


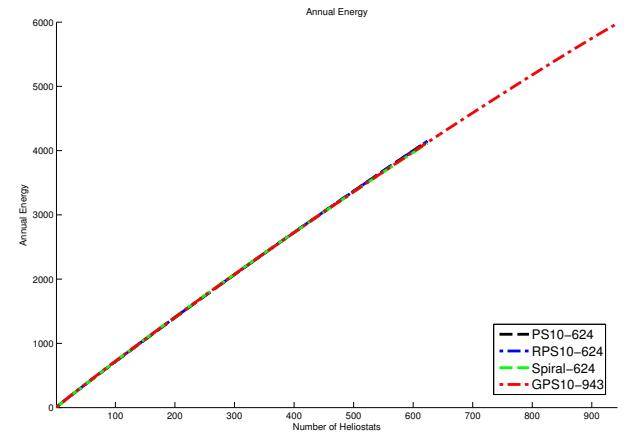

(a) Annual Thermal Energy per hel.

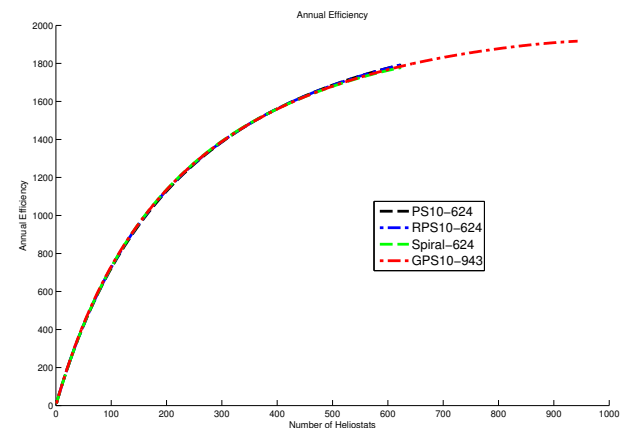

(c) Annual thermal Energy per unit cost per hel.

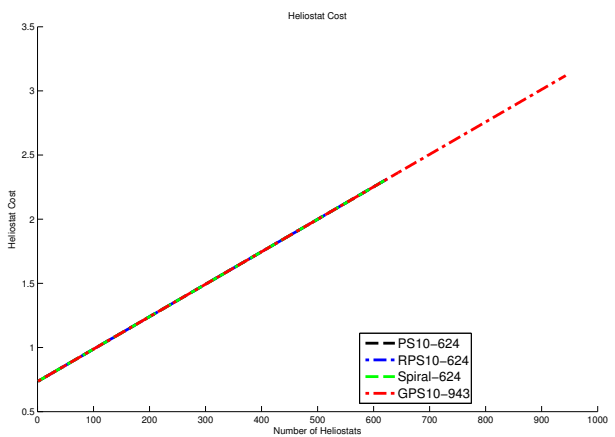

(b) Cost per heliostat

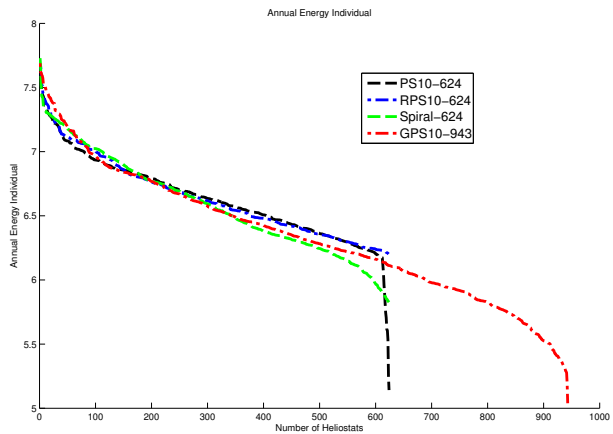

(d) Marginal Annual Thermal Energy

Figure 6: Field Layouts Analysis

The multimodality of the problem is rather strong. In Figure 7, the field layouts obtained using the greedy algorithm without multistart procedure and with multistart procedure are shown. When the greedy algorithm is used without multistart procedure the heliostat field layouts obtained are not so regular. The use of different values for $N_{\text {ini }}$ and for $F_{\text {sep }}$ lead to different results and the best results are not necessary matched with the field regularity. The results obtained are strongly dependant on the parameter $F_{\text {sep }}$, and thus this parameter must be carefully tuned. 


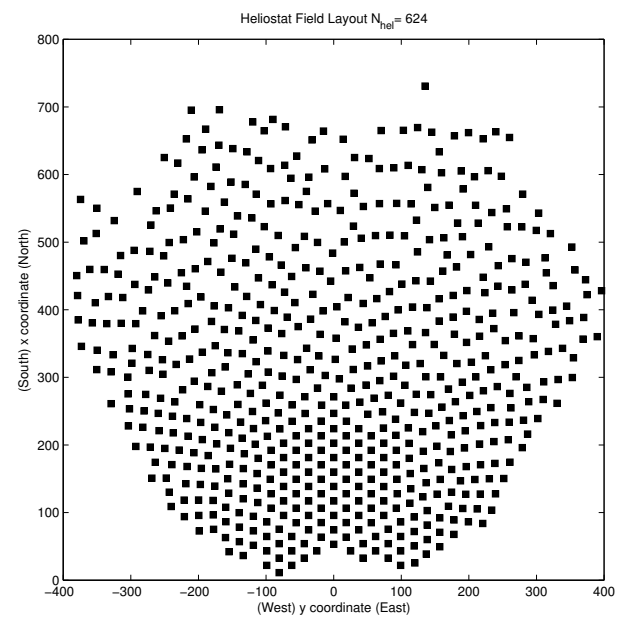

(a) $N_{\text {ini }}=1$

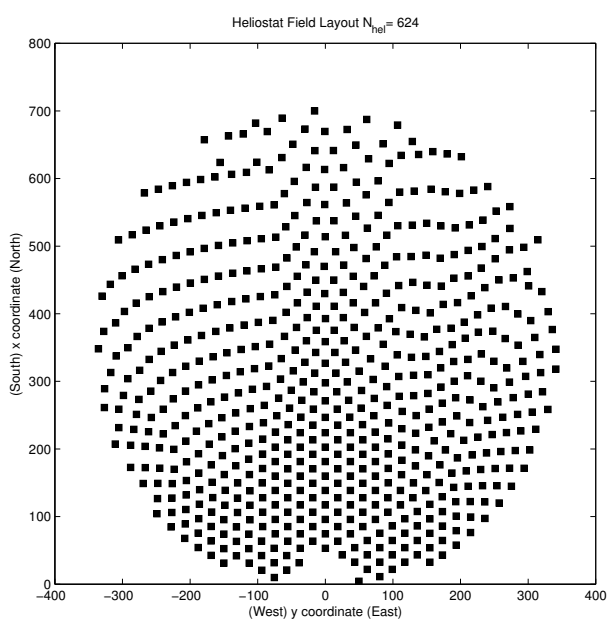

(b) $N_{\text {ini }}=25$

Figure 7: Multistart Analysis with $F_{\text {sep }}=1.4$

\subsection{Alternating procedure}

When the tower variables are also optimized, using our alternating approach we obtain step-by-step the results shown in Table 4 and Figure 8. Using random feasible values as initial data for the tower configuration and the Alternating Algorithm 1, a more efficient SPT system is sought. To make a fair comparison, the receiver outlet thermal energy at $T_{d}$ is fixed at 42.52 MWth, the same as the value obtained with the PS10 configuration. The parameter $N_{\text {ini }}$ is set to 25 , since this value gave the best results in some preliminary tests performed and $\epsilon$ is set to 0.01 . 


\begin{tabular}{|c|c|c|c|c|c|c|c|c|}
\hline Step & Problem & $\Theta_{k}$ & Fig. & $|\mathcal{S}|$ & $\Pi_{T_{d}}\left(\Theta_{k}, \mathcal{S}_{k}\right)$ & $E\left(\Theta_{k}, \mathcal{S}_{k}\right)$ & $F\left(\Theta_{k}, \mathcal{S}_{k}\right)$ & $F_{s e p}$ \\
\hline \multirow{2}{*}{$\mathrm{k}=0$} & Random $\Theta^{0}$ & $(13.01,30.16)$ & - & 0 & - & - & - & - \\
\hline & $\mathcal{S}_{0}:$ Solve $\left(\mathcal{P}_{\Theta}\right)$ & $(13.01,30.16)$ & $8(a)$ & 477 & 0.43 & 0.12 & 0.31 & 2.0 \\
\hline \multicolumn{9}{|c|}{$\Upsilon_{\text {objective }}=0.31$} \\
\hline \multicolumn{9}{|c|}{$\Upsilon_{\text {design }}=\left(\Theta^{0}, \mathcal{S}^{0}\right)$} \\
\hline \multirow{2}{*}{$\mathrm{k}=1$} & $\Theta_{1}:$ Solve $\left(\mathcal{P}_{\mathcal{S}}\right)$ & $(5.81,84.22)$ & - & 777 & 0.47 & 0.12 & 0.48 & 2.0 \\
\hline & $\mathcal{S}_{1}:$ Solve $\left(\mathcal{P}_{\Theta}\right)$ & $(5.81,84.22)$ & $8(b)$ & 633 & 0.43 & 0.12 & 0.53 & 1.6 \\
\hline \multicolumn{9}{|c|}{$\Upsilon_{\text {objective }}=0.53$} \\
\hline \multicolumn{9}{|c|}{$\Upsilon_{\text {design }}=\left(\Theta^{1}, \mathcal{S}^{1}\right)$} \\
\hline \multirow{2}{*}{$\mathrm{k}=2$} & $\Theta_{2}:$ Solve $\left(\mathcal{P}_{\mathcal{S}}\right)$ & $(6.05,87)$ & - & 633 & 0.45 & 0.12 & 0.52 & 1.6 \\
\hline & $\mathcal{S}_{2}:$ Solve $\left(\mathcal{P}_{\Theta}\right)$ & $(6.05,87)$ & $8(c)$ & 631 & 0.43 & 0.12 & 0.52 & 1.6 \\
\hline \multicolumn{9}{|c|}{$\Upsilon_{\text {objective }}=0.53$} \\
\hline \multicolumn{9}{|c|}{$\boldsymbol{\Upsilon}_{\text {design }}=\left(\Theta_{1}, \mathcal{S}_{1}\right)$} \\
\hline
\end{tabular}

Table 4: Results obtained using the Alternating Algorithm

From Table 4 we conclude that the best solution $\left(\Theta_{1}, \mathcal{S}_{1}\right)$ found at the second step of the Alternating Algorithm, collects a higher annual thermal energy per unit cost than the values of the three reference SPT systems. Note that the first heliostat field obtained in this example, see Figure 8(a), is not as compact as the heliostat fields obtained in next iterations. This effect is due to the low tower height value and the large receiver radius value obtained as initial random solution. When the alternating process continues this effect is corrected by the own algorithm. 


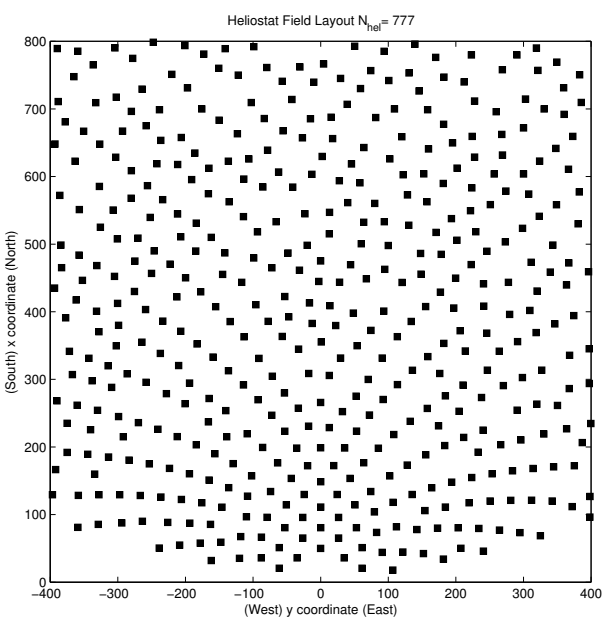

(a) $\mathcal{S}_{0}$

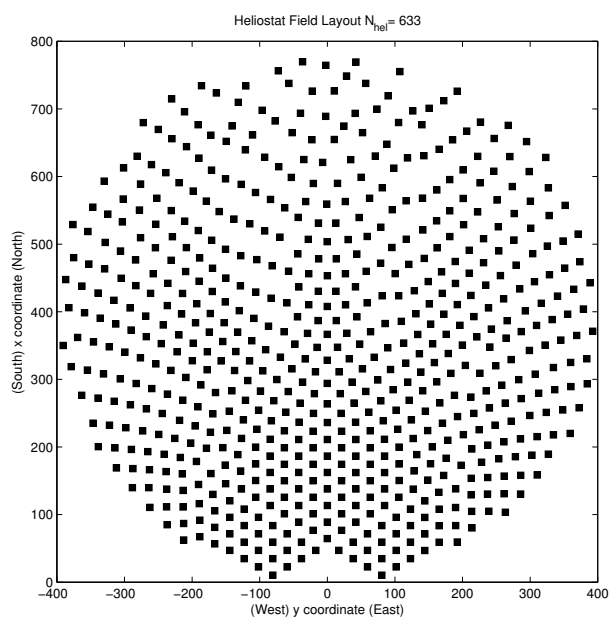

(b) $\mathcal{S}_{1}$

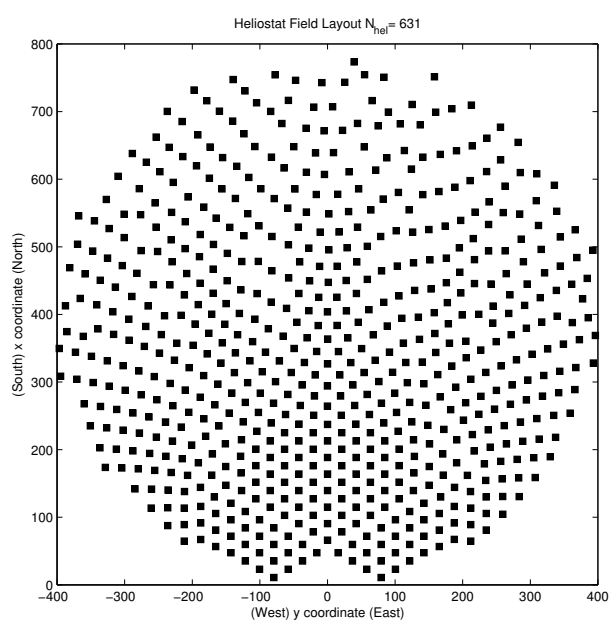

(c) $\mathcal{S}_{2}$

Figure 8: Field Layouts obtained using Algorithm 1

Using the alternating procedure, we cannot prove that it converges. However, as can be seen in Table 4, starting from a "bad solution" (low tower and big aperture), the algorithm leads to a very reasonable solution compared against the literature. The alternating algorithm could be embedded 
in a multistart process, starting with different random initial solution $\Theta_{0}$ and selecting as solution the best obtained configuration.

\subsection{Different feasible regions}

In real situations, the region selected to build the SPT system can have some terrain constraints. This means that some unfeasible zones need to be excluded of the original feasible region. Using the field layout algorithm described in Algorithm 3, different regions can be considered. The heliostat location procedure remains exactly the same, since with our algorithm the heliostat distribution is not parameterized, and the algorithm can adapt automatically the heliostat location to the selected region shape.

To illustrate the advantages of our pattern-free method against patternbased methods, three different possible feasible regions are considered and both procedures, radially-staggered parameterization and greedy algorithm are compared in these regions. The three feasible regions that are considered are: a rectangular region $(\mathrm{R})$, a perforated region $(\mathrm{P})$ and a valley region $(\mathrm{V})$, where the SPT system is supposed to be located near a river (see Figure 9). 


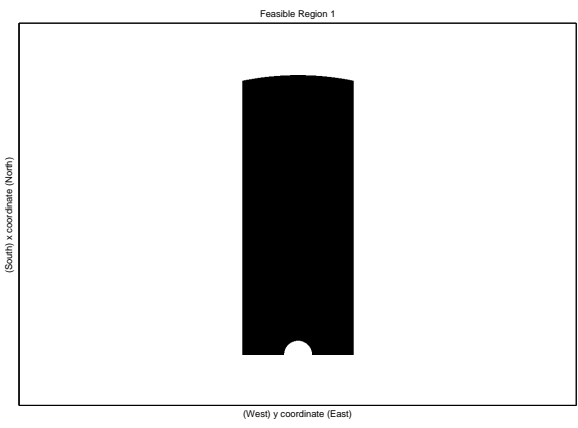

(a) Rectangular region (R)

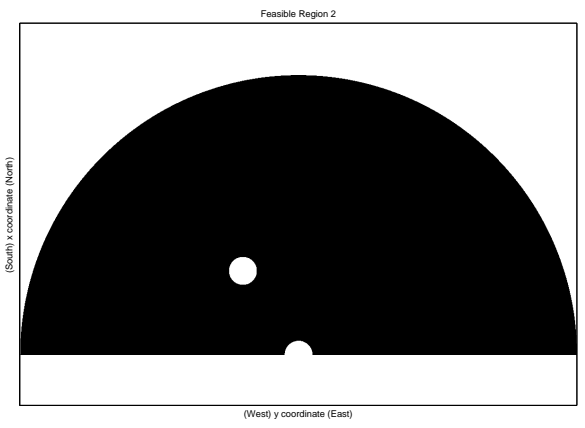

(b) Perforated region $(\mathrm{P})$

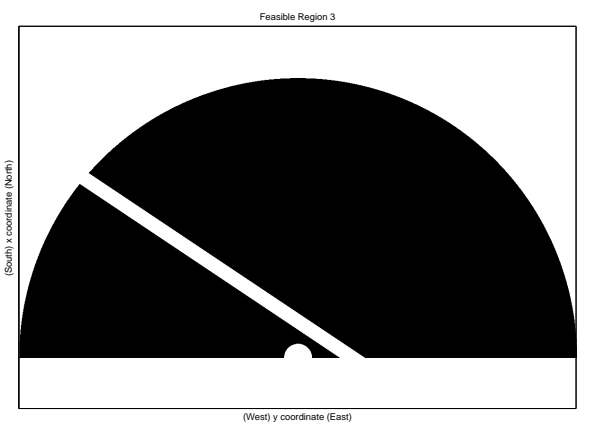

(c) Valley region $(\mathrm{V})$

Figure 9: Feasible Regions

As said before, we design the heliostat field for each feasible region considering the receiver outlet thermal energy at $T_{d}$ as $\Pi_{0}=42.52 \mathrm{MWth}$. The fields are obtained using Algorithm 3 and for each feasible region the different constraints associated are considered. In order to compare these fields, we have limited the PS10 and RPS10 configurations to the three different regions and evaluated the results.

In Figures 10-12, the heliostat fields for each feasible regions are shown. The different phases of the greedy algorithm, Phase A and Phase B, are detailed numerically in Table 5. From the results we conclude that for most 
tests performed the annual thermal energy collected per unit cost improves with our greedy based procedure, and in the case that this value is not improved we propose a similar solution.

Fixed-pattern algorithms ignore the shape of the feasible field region and are always limited to the geometrical pattern selected. By contrast, in Figure 11, the adaptation of the heliostat positions through the greedy algorithm around the circle perforation can be appreciate. Also, using our pattern-free procedure the density of the field is automatically adapted to the field characteristics and takes advantage of the best region. These local adaptations are not automatically done using the original radially-staggered pattern. 


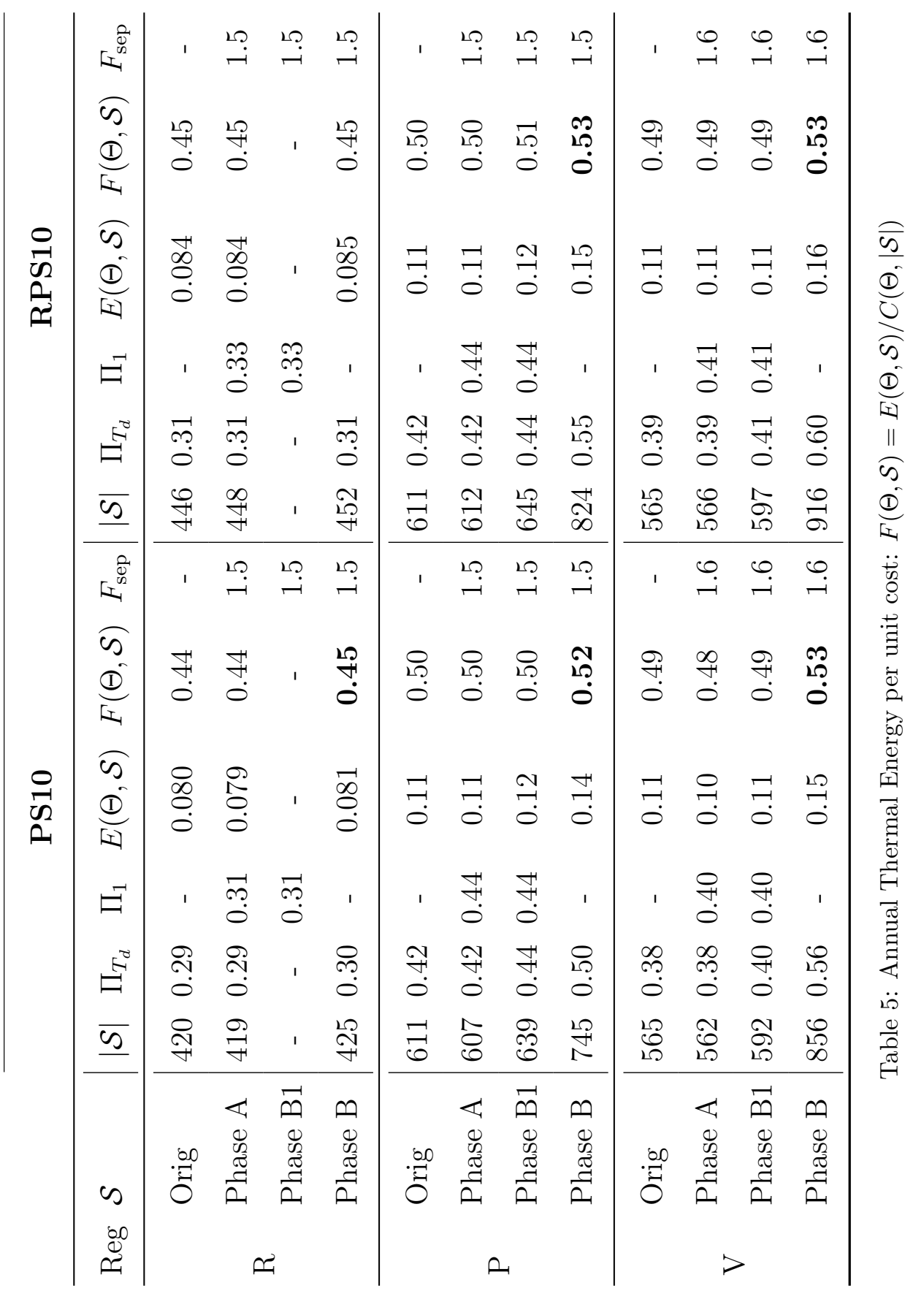



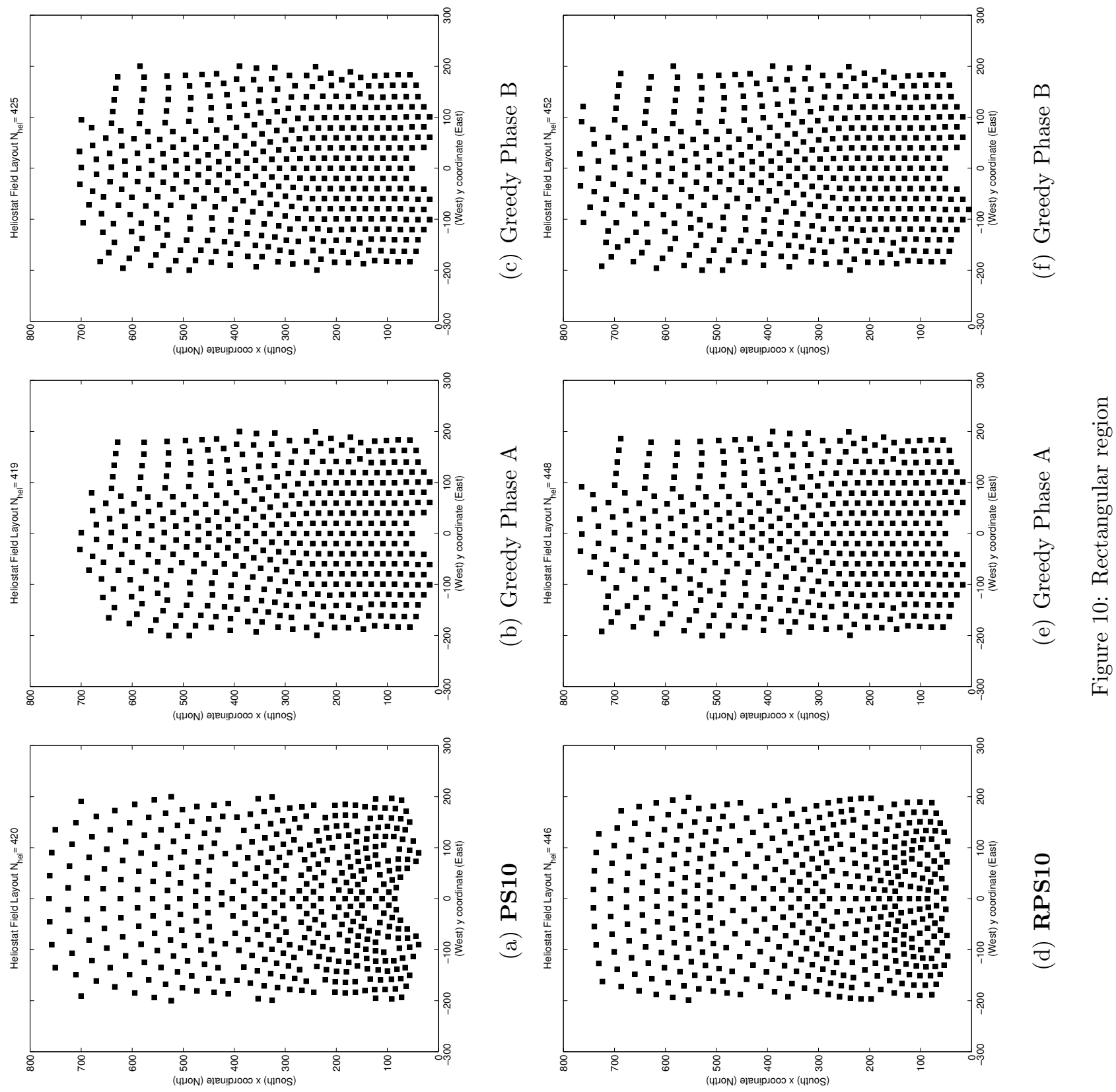

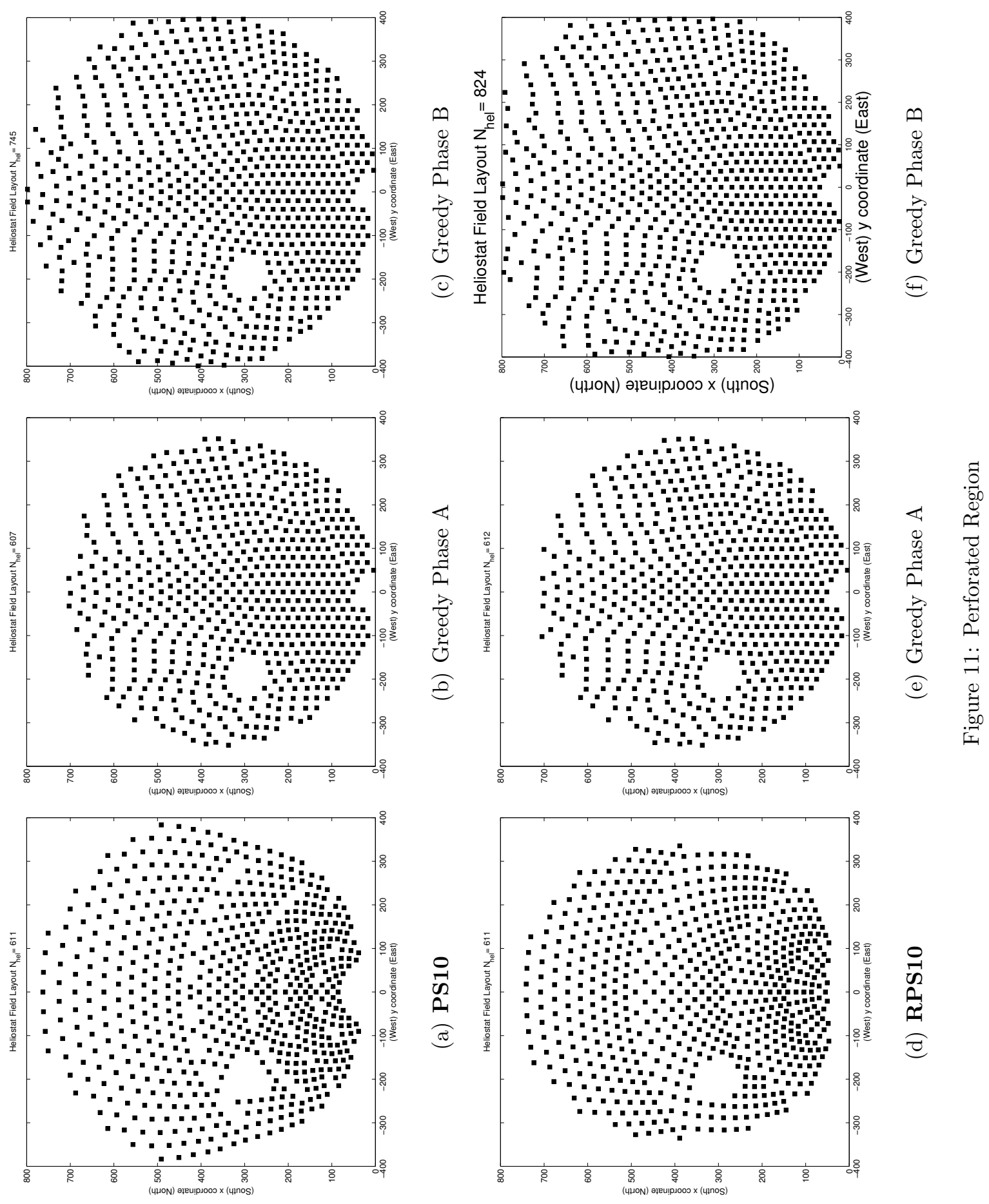

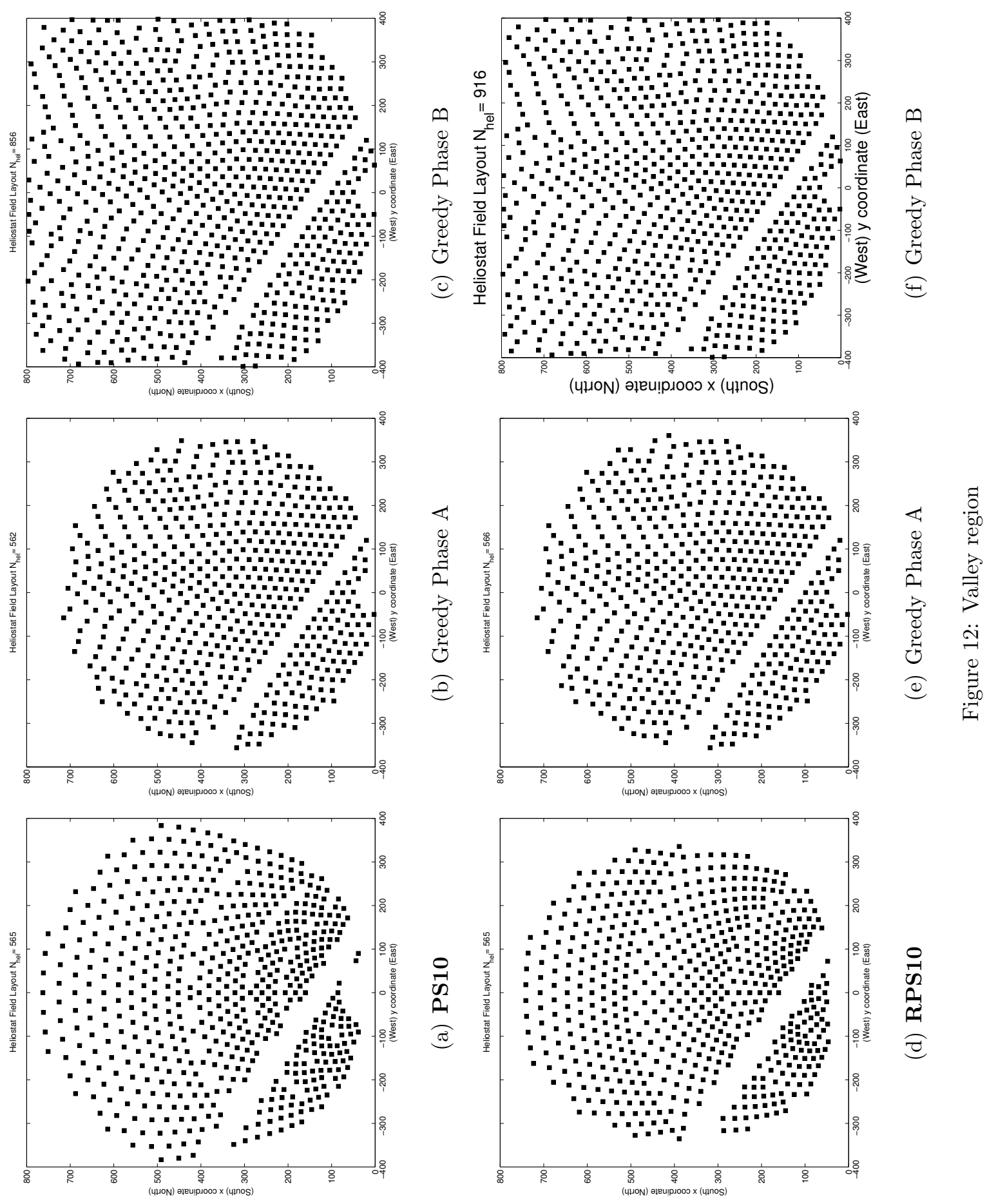


\section{Conclusions}

A pattern-free method for optimizing an SPT system has been proposed, in which both the location of the heliostats and the design of the solar tower are simultaneously considered. Maximizing the thermal energy collected per unit cost leads to a difficult optimization problem, since it has nonconvex constraints and a nonconvex objective function, given in a black-box form which is computationally expensive to evaluate.

An alternating greedy-based heuristic has been suggested. Our method provides competitive results against the standard results in the literature and is more versatile since it is not based on geometrical patterns which may be valid only under certain physical conditions.

For simplicity, in this article we consider that the heliostat size is fixed, however our procedure can be extended to consider it as an optimization variable. In Carrizosa et al. (2014), we address the problem of heliostat field design considering two different sizes. Note that using a fixed-pattern approach, this new problem cannot be easily solved as a different pattern configuration must be studied. Considering also different receiver technologies, as multiple receivers with different fields aiming to each receiver is also an interesting problem to be studied. Furthermore, our strategy can be used to obtain an initial field for other algorithms (such as the one in Buck et al. (2012)), and can be extended to address many other situations: ground irregularities in the field, the effect of tower shading, variable (stochastic) meteorological data and Multi-Tower plants.

Unfortunately, given the complexity of the model and our heuristic methodology, it is not possible to easily perform an error analysis. However, using 
simpler models, having a low number of heliostats, the quality of the solutions could be measured. As already mentioned, when considering a reduced number of heliostats a simultaneous heliostat location could be possible. This is a very interesting problem to be addressed in the future.

Other different challenging problems are mentioned below. The design of procedures able to approximate the efficient frontier for the biobjective problem (maximizing thermal energy collected and minimizing cost). The recent work Spelling et al. (2012) is a promising first approach. The study of the energy storage operations (see Ghobeity \& Mitsos (2012); Sheu et al. (2012)) and the study of aiming strategies (see Belhomme et al. (2014)).

\section{Acknowledgements}

The authors thank Noone et al. (2012) for providing the heliostat field configurations used to compare the results.

This research has been mainly supported by Abengoa Solar N.T. and Institute of Mathematics of University of Seville (IMUS), through the research contract "CapTorSol". The authors would also like to acknowledge the support from the Governments of Spain (Grants MTM2010-15992, MTM201236136), Andalucía (Grant P11-FQM-7603) and EU COST Action TD1207.

\section{References}

Alon, L., Ravikovich, G., Mandelbrod, M., Eilat, U., Schop, Z., \& Tamari, D. (2014). Computer-based management of mirror-washing in utility-scale solar thermal plants. In Proceedings in ASME 2014 8th International Conference on Energy Sustainability collocated with the ASME 2014 12th In- 
ternational Conference on Fuel Cell Science, Engineering and Technology. volume 1 .

Bazaraa, M. S., Sherali, H. D., \& Shetty, C. M. (2006). Nonlinear Programming: Theory and Algorithms. John Wiley and Sons, Hoboken, New Jersey.

Behar, O., Khellaf, A., \& Mohammedi, K. (2013). A review of studies on central receiver solar thermal power plants. Renewable and Sustainable Energy Reviews, 23, 12-39.

Belhomme, B., Pitz-Paal, R., \& Schwarzbözl, P. (2014). Optimization of heliostat aim point selection for central receiver systems based on the ant colony optimization metaheuristic. Journal of Solar Energy Engineering, $136,1-7$.

Biggs, F., \& Vittitoe, C. (1976). The HELIOS model for the optical behavior of reflecting solar concentrators. Technical Report SAND76-0347 Sandia National Labs. URL: http://prod.sandia.gov/techlib/access-control.cgi/1976/760347.pdf.

BrightSource (2014). Ivanpah Solar Electric Generating System. Technical Report BrightSource. URL: http://www . brightsourceenergy . com/stuff/contentmgr/files /0/3eac1a9fed7f13fe4006aaab8c088277/attachment/ ivanpah_white_paper_0414.pdf.

Buck, R. (2014). Heliostat field layout improvement by nonrestricted refinement. Journal of Solar Energy Engineering, 136, 1-6. 
Buck, R., Barth, C., Eck, M., \& Steinmann, W. (2006). Dual receiver concept for solar tower. Solar Energy, 80, 1249-1254.

Buck, R., Pfahl, A., \& Roos, T. H. (2012). Target aligned heliostat field layout for non-linear flat terrain. In Proceedings of the First Southern African Solar Energy Conference (SASEC 2012).

Burgaleta, J. I., Arias, S., \& Ramirez, D. (2011). GEMASOLAR, the first tower thermosolar commercial plant with molten salt storage. In Proceedings of SolarPaces 2011.

Carrizosa, E., Domínguez-Bravo, C., Fernández-Cara, E., \& Quero, M. (2014). An Optimization Approach to the Design of Multi-Size Heliostat fields. Technical Report IMUS. URL: http://www.optimization-online.org/DB_HTML/2014/05/4372.html.

Collado, F. J. (2008). Quick evaluation of the annual heliostat field efficiency. Solar Energy, 82, 379-384.

Collado, F. J. (2009). Preliminary design of surrounding heliostat fields. Renewable Energy, 34, 1359-1363.

Collado, F. J., \& Guallar, J. (2012). Campo: Generation of regular heliostat fields. Renewable Energy, 46, 49-59.

Collado, F. J., \& Turégano, J. (1986). An analytic function for the flux density due to sunlight reflected from a heliostat. Solar Energy, 37, 215234. 
Collado, F. J., \& Turégano, J. (1989). Calculation of the annual thermal energy supplied by a defined heliostat field. Solar Energy, 42, 149-165.

Crespo, L., \& Ramos, F. (2009). NSPOC: A New Powerful Tool for Heliostat Field Layout and Receiver Geometry Optimizations. In Proceedings of SolarPaces 2009.

Ghobeity, A., \& Mitsos, A. (2012). Optimal Design and Operation of a Solar Energy Receiver and Storage. Solar Energy Engineering, 134, 9 pages.

Lipps, F. W., \& Vant-Hull, L. L. (1978). A cellwise method for the optimization of large central receiver systems. Solar Energy, 20, 505-516.

Mills, D. R. (2004). Advances in solar thermal electricity technology. Solar Energy, 76, 19-31.

Noone, C. J., Torrilhon, M., \& Mitsos, A. (2012). Heliostat field optimization: A new computationally efficient model and biomimetic layout. Solar Energy, 86, 792-803.

Osuna, R., Fernández, V., Romero, S., Romero, M., \& Sánchez, M. (2004). PS10: a 11.0-MWe Solar Tower Power Plant with Satured Steam Receiver. In Proceedings of SolarPaces 2004. Editors: C. Ramos and J. Huacuz.

Pitz-Paal, R., Botero, N. B., \& Steinfeld, A. (2011). Heliostat field layout optimization for high-temperature solar thermochemical processing. Solar Energy, 85, 334-343.

Ramos, A., \& Ramos, F. (2012). Strategies in Tower Solar Power Plant optimization. Solar Energy, 86, 2536-2548. 
Romero, M., Buck, R., \& Pacheco, J. E. (2002). An update on Solar Central Receiver Systems, Projects and Technologies. Solar Energy Engineering, 124, 98-109.

Sánchez, M., \& Romero, M. (2006). Methodology for generation of heliostat field layout in central receiver systems based on yearly normalized energy surfaces. Solar Energy, 80, 861-874.

Sanz-Bermejo, J., Gallardo-Natividad, V., Gonzalez-Aguilar, J., \& Romero, M. (2014). Comparative system performance analysis of direct steam generation central receiver solar thermal power plants in megawatt range. Journal of Solar Energy Engineering, 136, 1-9.

Sassi, G. (1983). Some notes on shadow and blockage effects. Solar Energy, 31, 331-333.

Sheu, E. J., Mitsos, A. A., A. Eter, Mokheimer, E. M. A., Habib, M. A., \& AlQutub, A. (2012). A Review of Hybrid Solar-Fossil Fuel Power Generation Systems and Performance Metrics. Journal of Solar Energy Engineering, 134, 17 Pages.

Siala, F. M. F., \& Elayeb, M. E. (2001). Mathematical formulation of a graphical method for a no blocking heliostat field layout. Renewable Energy, 23, 77-92.

Spelling, J., Favrat, D., Martin, A., \& Augsburger, G. (2012). Thermoeconomic optimization of a combined-cycle solar tower power plant. Energy, 41, 113-120. 
Stine, W. B., \& Harrigan, R. W. (2001). Power From The Sun. John Wiley and Sons. URL: http://www. powerfromthesun.net/book.html.

Wei, X., Lu, Z., Wang, Z., Yu, W., Zhang, H., \& Yao, Z. (2010). A new method for the design of the heliostat field layout for solar tower power plant. Renewable Energy, 35, 1970-1975.

Zhang, H., Juchi, I., Favrat, D., \& Pelet, X. (2007). Multi-objective thermoeconomic optimisation of the design of heliostat field of solar tower power plants. In Proceedings of the Engineering for sustainable Energy in developing countries. 